\title{
The Level of Care Needs of Patients With Cancer and the Time-dependent Burden on Their Family Caregivers in Home Palliative Care: A Cross- Sectional Study
}

\section{Naoko Otsuki}

Osaka University: Osaka Daigaku

Ryohei Yamamoto ( $\square$ yamamoto@hacc.osaka-u.ac.jp)

Osaka University: Osaka Daigaku https://orcid.org/0000-0003-2610-9824

Yukihiro Sakaguchi

Kwansei Gakuin University: Kansei Gakuin Daigaku

Kento Masukawa

Tohoku University: Tohoku Daigaku

Tatsuya Morita

Seirei Mikatahara Hospital: Seirei Mikatahara Byoin

Yoshiyuki Kizawa

Kobe University: Kobe Daigaku

Satoru Tsuneto

Kyoto University: Kyoto Daigaku

Yasuo Shima

Tsukuba Medical Center Hospital

Sakiko Fukui

Tokyo Medical and Dental University: Tokyo Ika Shika Daigaku

Mitsunori Miyashita

Tohoku University: Tohoku Daigaku

\section{Research Article}

Keywords: care needs level, burden, home palliative care, family caregiver, patient with cancer

Posted Date: May 27th, 2021

DOI: https://doi.org/10.21203/rs.3.rs-431425/v1

License: (c) (i) This work is licensed under a Creative Commons Attribution 4.0 International License. 



\section{Title page}

Title: The level of care needs of patients with cancer and the time-dependent burden on their family caregivers in home palliative care: A cross-sectional study

Authors: Naoko Otsuki, RN, MS, ${ }^{1}$ Ryohei Yamamoto, MD, PhD, ${ }^{2}$ Yukihiro

Sakaguchi, $\mathrm{PhD},{ }^{3}$ Kento Masukawa, RN, MS, ${ }^{4}$ Tatsuya Morita, MD, PhD,${ }^{5}$ Yoshiyuki

Kizawa, MD, $\mathrm{PhD},{ }^{6}$ Satoru Tsuneto, $\mathrm{MD}, \mathrm{PhD},{ }^{7}$ Yasuo Shima, $\mathrm{MD}, \mathrm{PhD},{ }^{8}$ Sakiko

Fukui, RN, $\mathrm{PhD},{ }^{9}$ and Mitsunori Miyashita $\mathrm{RN}, \mathrm{PhD},{ }^{10}$

\section{Affiliations:}

${ }^{1}$ Division of Health Science, Osaka University Graduate School of Medicine. 1-7

Yamadaoka, Suita, Osaka, JAPAN. E-mail: otsuki.chn@tmd.ac.jp

${ }^{2}$ Health and Counseling Center, Osaka University, 1-17 Machikaneyama, Toyonaka,

Osaka, JAPAN. Department of Nephrology, Osaka University Graduate School of

Medicine. 2-2 Yamadaoka, Suita, Osaka, JAPAN. Health Promotion and Regulation,

Department of Health Promotion Medicine, Osaka University Graduate School of

Medicine. 2-2 Yamadaoka, Suita, Osaka, JAPAN.

E-mail: yamamoto@hacc.osaka-u.ac.jp 
${ }^{3}$ Kwansei Gakuin University School of Human Welfare Studies. 1-1-155, Uegahara,

Nishinomiya, JAPAN. E-mail: sakaguchi@kwansei.ac.jp

${ }^{4}$ Department of Palliative Nursing, Health Sciences, Tohoku University Graduate

School of Medicine. 2-1 Seiryo-machi, Aoba-ku, Sendai, JAPAN.

E-mail: kmasukawa.24@med.tohoku.ac.jp

${ }^{5}$ Division of Palliative and Supportive Care, Seirei Mikatahara Hospital. 3453

Mikatahara, Kita, Hamamatsu, JAPAN. E-mail: tmorita@sis.seirei.or.jp

${ }^{6}$ Kobe University Graduate School of Medicine. 7-5-1, Kusunoki-cho, Chuo-ku, Kobe,

JAPAN. E-mail: ykizawa@med.kobe-u.ac.jp

${ }^{7}$ Department of Human Health Sciences, Kyoto University Graduate School of

Medicine. 53 Kawaharacho, Shogoin, Sakyo-ku, Kyoto, JAPAN.

E-mail: tsuneto@kuhp.kyoto-u.ac.jp

${ }^{8}$ Department of Palliative Medicine, Tsukuba Medical Center Hospital. 1-3-1,

Amakubo, Tsukuba, Ibaragi, JAPAN. E-mail: shima@tmch.or.jp

${ }^{9}$ Tokyo Medical and Dental University Graduate School of Health Care Sciences. 1-5-

45, Yushima, Bunkyo-ku, Tokyo, JAPAN. E-mail: fukuisakiko.chn@tmd.ac.jp

${ }^{10}$ Department of Palliative Nursing, Health Sciences, Tohoku University Graduate

School of Medicine. 2-1 Seiryo-machi, Aoba-ku, Sendai, JAPAN. 
E-mail: miya@med.tohoku.ac.jp

Corresponding author: Ryohei Yamamoto, MD, PhD

Affiliation: Health and Counseling Center, Osaka University

Address: 1-17 Machikaneyama, Toyonaka, Osaka, 560-0043 JAPAN

E-mail: yamamoto@hacc.osaka-u.ac.jp

TEL: +81-6-6850-6015

\begin{abstract}
Purpose: Although home care improves patients' quality of life (QOL), several studies have suggested that home care lowers the QOL and mortality of family caregivers. To alleviate the deleterious impact of home care on caregivers, the major burdens on caregivers and the clinical characteristics of the caregivers vulnerable to the major burden needs to be clarified.
\end{abstract}

Method: A survey questionnaire was distributed to 710 family caregivers of patients with cancer in Japan, and 342 valid responses were obtained (valid response rate: 48.2\%). The Burden Index of Caregivers was used to identify the major burden on caregivers. To assess the associations of the patients' care needs level and other 
clinically relevant factors with the major burden, a multivariable-adjusted logistic regression model was used.

Results: The time-dependent burden was identified as a major burden. An adjusted model showed a non-linear association between the care needs level and the timedependent burden, in which the caregivers of the patients who required moderate care needs level had the highest time-dependent burden [adjusted odds ratio of none, mild, moderate, and severe care needs levels: 0.50 (95\% confidence interval 0.07-2.12), 1.08 (0.43-2.57), 1.87 (1.01-3.52), and 1.00 (reference), respectively]. Additionally, older patients and younger caregivers were significantly associated with a time-dependent burden.

Conclusion: The time-dependent burden was highest in caregivers at the moderate care needs level and younger caregivers. An imbalance between the demand and supply of care services may be improved by considering the clinical characteristics of both patients and caregivers.

Keywords: care needs level, burden, home palliative care, family caregiver, patient with cancer. 


\section{Declarations}

Funding support: This study was conducted as a specific research of the JHOPE4 study funded by the Japan Hospice Palliative Care Foundation and JSPS KAKENHI Grant Number JP18J225780.

Conflict of interest disclosures: The authors declare no conflict of interest.

Availability of data: The data were collected by questionnaire with the approval of the Ethics Committee of Tohoku University.

Code availability: N/A

Author contributions: Conceptualization: [N. Otsuki, R. Yamamoto, Y. Sakaguchi], Methodology: [N. Otsuki, R. Yamamoto], Formal analysis: [N. Otsuki, R. Yamamoto], Investigation: [K. Masukawa, T. Morita, Y. Kizawa, S. Tsuneto, Y. Shima, M.

Miyashita], Writing - original draft preparation: [N. Otsuki, R. Yamamoto]; Writing review and editing: [R. Yamamoto, Y. Sakaguchi, S. Fukui], Funding acquisition: [N. Otsuki, M. Miyashita], Resources: [M. Miyashita], Supervision: [S. Fukui], Project administration: [M. Miyashita]

Ethics approval: The study protocol of JHOPE was approved by the ethics committee of Tohoku University Hospital and all participating institutions, and the protocol of the present study was approved by the Osaka University Hospital. 
All family caregivers gave informed consent to JHOPE4.

Consent for participation and publication: Informed consent was given to all family caregivers for participation in JHOPE4 and possible publication of the results.

Acknowledgments: None 


\section{Introduction}

The aging society has increased the number of people requiring advanced medical care in many developed countries[1]. In Japan, rapid population aging since 1970 has produced the world's leading super-aging society[2]. To support the aging society in Japan, home care has been primarily promoted in the long-term care insurance system established in 2000[3-5], because home care improves patients' quality of life (QOL)[6]. However, previous studies have reported that family caregivers had a high mortality rate and low QOL, suggesting that home care might have detrimental effects on family caregivers. The self-perceived burden is a potential determinant of the effect of home care on family caregivers because family caregivers with mental strain had a higher mortality rate than those without mental strain[7] and the self-perceived burden was associated with the QOL of family caregivers[8].

To develop an effective strategy to alleviate the deleterious impact of home care on family caregivers, the major domains of the burden on family caregivers should be clarified before considering how to support them. Although multiple studies have reported the overall burden on family caregivers, commonly using the Caregiver Reaction Assessment (CRA), the Zarit Burden Inventory, and the Caregiver Burden Index, few studies have reported the major domain of the burden on family caregivers. 
Among five domains of the CRA that include the impact of schedule, caregiver's esteem, lack of family support, impact on health, and impact on finances[9], caregivers' esteem was identified as the major burden on family caregivers of patients with lung cancer in the US $(n=158)[10]$ and those with multiple cancers in Norway $(n=281)[3]$, while the impact of schedule was identified as the major burden on family caregivers of patients with lung cancer in Taiwan $(\mathrm{n}=150)[11]$. To identify the family caregivers at a high risk of the major burden, clinical characteristics of the patients and the family caregivers associated with the major burden are very useful, although previous studies did not report them[3, 10,11]. One of the potential factors associated with the major burden may be patients' functional status because multiple studies have reported a linear dose-dependent association between the patients' functional status and the overall burden on the family caregivers[3, $8,12,13]$.

The aims of the present cross-sectional study that included 342 family caregivers of patients receiving home palliative care for multiple cancers in Japan, were, first, to identify the major burden on the family caregivers and, second, to clarify the clinical characteristics of the caregivers who were vulnerable to the major burden. The results of the present study could provide clinically useful evidence to establish an 
effective strategy to alleviate the major burden on caregivers of patients receiving home palliative care for cancer in the Japanese home care system.

\section{Materials and Methods}

\section{Study population}

The Japan Hospice and Palliative Evaluation Study 4 (JHOPE4) is a cross-

sectional study that assesses the quality of palliative care for patients with cancer using the information provided by their bereaved family caregivers. JHOPE4 included 17,147 adult patients aged $\geq 20$ years with palliative care of $\geq 3$ days who died of cancer between 2014 and 2017 and their main family caregivers. JHOPE4 collected information from the family caregivers on the physical and mental status of both patients and their family caregivers, the quality of palliative care evaluated by the family caregivers, and the burden on the family caregivers[14]. To assess the burden on the family caregivers who cared for patients who died at home, the present study included 1,050 family caregivers of patients who received home palliative care and died at home, after excluding 16,097 family caregivers of patients who died in acute hospitals or at inpatient palliative care units. Based on the pre-specified research questions, 1,050 family caregivers were grouped into three clusters. Questionnaires used 
in the present study were sent to $710(67.6 \%)$ family caregivers in clusters 1 and 2 , and 495 (47.1\%) family caregivers responded. After excluding 153 (14.6\%) family caregivers with missing data, the present study finally included $342(32.6 \%)$ family caregivers of patients with cancer.

\section{Measurements}

The clinical characteristics of the patients with cancer during the home

palliative care period included the age, sex, type of cancer, home palliative care period, and care needs level certified at the end of the home care period. The types of cancer were classified into four groups as follows: lung; upper and lower gastrointestinal tracts including esophageal, gastric, and colorectal cancer; liver, biliary tract, and pancreas; and others[15]. Care needs level was based on the degree of care required as by the patients per the Japanese long-term care insurance system, consisting of 7 levels as follows: requiring support 1 and 2 and requiring long-term care 1, 2, 3, 4, and 5[1, 4, 5, $16,17]$. The care needs level is determined according to the patient's physical and mental conditions with activities of daily living and instrumental activities of daily living, and physicians' medical diagnoses and generally updated every 6-12 months[5, 17]. The care needs level is divided into requiring support and requiring long-term care, in which requiring support provides preventive benefits and requiring long-term care 
provides long-term benefits[18]. According to the patients' care needs levels, we categorized 342 family caregivers into the following four groups because of a limited number of the patients in some levels: Mild, requiring support $1(n=13)$ and $2(n=15)$ and requiring long-term care $1(n=24)$; Moderate, requiring long-term care $2(n=65)$ and $3(n=60)$; Severe, requiring long-term care $4(n=74)$ and $5(n=69)$; and None, no certification for long-term care $(\mathrm{n}=22)$. We combined requiring support $1-2$ and requiring long-term care 1 into the mild group because the patients at these levels had similar functional status[19].

The information that was collected from family caregivers included age, sex, relationship with the patients, mental condition, and self-perceived burden during the home care period. The self-perceived burden on the family caregivers was measured using the Burden Index of Caregivers (BIC) questionnaire[20] that included ten questions in five domains (time-dependent, emotional, spiritual, physical, and servicerelated burden) and a single question for total burden. For example, the time-dependent domain includes two questions as follows: "I cannot freely leave the house because of caregiving" and "I do not have enough time for myself because of caregiving" with 5 possible answers of "never (score 0)," "almost never (1)," "sometimes (2)," "often (3)," and "always (4)." Other questions with the same five possible answers are listed in 
Supporting Table 1. The present study used ten questions in five domains of caregiving to assess the clinical impact of each domain on family caregivers. The score of each domain was the sum of two component questions (score $0-4$ ) with a range of $0-8$. The family caregivers had a burden for a domain if they answered "often" or "always" to at least one of two questions related to the domain. The mental status of the caregiver was determined by a question "While the patient was under care, how was your mental health condition?" with four possible answers of "good (score 1)," "average (2)," "bad (3)," and "very bad (4)."

\section{Statistical analyses}

The clinical characteristics of the patients with cancer and their family caregivers were compared among the four groups of the care needs level, using the chisquared test and the Kruskal-Wallis test, as appropriate.

To clarify the association between the patients' care needs level and the caregivers' burden, we first identified the major burden on the family caregivers among the five BIC domains, according to the proportion of the family caregivers with the burden of each domain. Second, to assess the association between the care needs level and the major burden, unadjusted and adjusted logistic regression models were used, including the major burden as an independent variable. Adjusted models included the 
age of patients and family caregivers, sex of patients and family caregivers, type of cancer of the patients, family caregivers' relationship with patients, the mental condition of family caregivers, and the home care period as independent variables. The HosmerLemeshow test was used to assess the goodness of fit of the logistic regression models. Continuous variables were expressed as mean \pm standard deviation or median $(25 \%-75 \%)$, as appropriate, and categorical variables were expressed as numbers (proportions). We used R statistical software, version 3.6.3, (R Development Core Team, 2020) for analysis. All statistical tests were two-sided and the values of $\mathrm{P}<0.05$ were considered statistically significant.

\section{Results}

The clinical characteristics of 342 included and 153 excluded patients with cancer and their family caregivers were compared, and it was found that the characteristics were comparable ( $\mathrm{P} \geq 0.05$ for all variables considered) (Supporting Table 2). The clinical characteristics of the 342 pairs stratified by care needs level are listed in Table 1. No significant differences were observed in all variables among 22 (6.4\%), $52(15.2 \%), 125(36.5 \%)$, and $143(41.8 \%)$ participants for the care needs level of none, mild, moderate, and severe, respectively, suggesting that their clinical 
characteristics were similar among the care needs levels. The median age of the patients for the care needs level of none, mild, moderate, and severe was found to be 73 (59-80), 76 (70-83), 78 (68-87), and 76 (68-84) years, respectively, and that of the family caregivers was found to be $65 \pm 11,65 \pm 12,62 \pm 12$, and $62 \pm 12$ years, respectively, indicating that the caregivers were approximately 10 years younger than participants.

To identify the major burden on family caregivers, the proportion of the answers to each BIC question is described in Figure 2. Because of the small number of caregivers who answered "always" and "often," they were categorized into a single $\geq$ often group. The proportion of the $\geq$ often group was $15.8 \%, 19.9 \%, 2.0 \%, 2.9 \%, 2.6 \%$, $2.3 \%, 5.8 \%, 4.1 \%, 3.2 \%$, and $0.9 \%$ in BIC questions $1-10$, respectively, indicating that the time-dependent domain consisting of questions 1 and 2 was a major burden for the family caregivers $[\mathrm{n}=77(22.5 \%)]$. To assess the dose-dependent association between the care needs level and the time-dependent burden, the proportion of the answers to BIC questions 1 and 2 in each care needs level is described in Figures 3a and 3b, respectively. In both questions, the proportion of the $\geq$ often group was found to be lowest in the none group ( $4.5 \%$ and $4.5 \%$ in questions 1 and 2 , respectively) and highest in the moderate care needs level (20.0\% and $28.0 \%$ in questions 1 and 2 , respectively). The time-dependent burden, defined as answering $\geq$ often to BIC question 1 and/or 2, 
was found to be least common in the none care needs level and most common in the moderate care needs level (Figure 3c), indicating a non-linear association between the care needs level and the time-dependent burden.

The association between the patients' care needs level and caregivers' timedependent burden was further assessed using the unadjusted and multivariable-adjusted logistic regression models, including the time-dependent burden as an independent variable. The unadjusted logistic regression models showed that the care needs level was significantly associated with the time-dependent domain [unadjusted odds ratio that was obtained for the none, mild, moderate, and severe care needs level: $0.43(0.07-$ 1.60), 1.02 (0.44-2.24), 1.88 (1.07-3.33), and 1.00 (reference), respectively), along with the age of patients and family caregivers (Table 2). Even after adjusting for the age and sex of patients and family caregivers, types of cancer, family caregivers' relationship with patients, mental status of family caregivers, home care period, the family caregivers and the patients at moderate care need level were found to have a significantly higher prevalence of the time-dependent domain, compared with those in patients with severe care needs level [0.50 (0.07-2.12), $1.08(0.43-2.57), 1.87(1.01-$ 3.52), and 1.00 (reference), respectively], ascertaining the non-linear association between the care needs level and the time-dependent burden. Besides the care needs 
level, older patients and younger caregivers were found to be significantly associated with the time-dependent burden as indicated in the multivariable-adjusted model.

\section{Discussion}

The present multicenter cross-sectional study that included 342 family caregivers with patients receiving home palliative care for multiple cancers, clarified that, first, the time-dependent burden was the major burden on the family caregivers and, second, the patients' care needs levels were associated with the time-dependent burden in a non-linear fashion.

Because we found a good correlation between the care needs level and the Barthel index, an indicator of the functional status of the patient (Pearson's $r=-0.70$ ), especially in patients aged $65-74$ years $(r=-0.80)$, the second finding of the present study strongly suggested that the patients' functional status was associated with the time-dependent burden in a non-linear fashion. Multiple studies have reported a linear dose-dependent association between patients' functional status and caregiver burden in Italy[8], US[12], Singapore[21], and South Korea[22]. The non-linear association that was found in the present study might indicate an imbalance between demand (care needs level) and supply (care provided) in the long-term care system in Japan, 
suggesting that family caregivers of patients with moderate care needs level (requiring long-term care 2-3) needed more support than those with severe care needs level. In Japan, based on the patients' care needs level, the long-term care system provides the patients who require a level of long-term care 1 with the light use of care services (home help, assistive device), those who require the level of long-term care 2 with day care with rehabilitation, those who require a level of long-term care 3 with short-stay respite care, and those who require the level of long-term care 4 and 5 with compound use of visiting services[23]. To improve the imbalance between the demand and supply in the Japanese long-term care system, more frequent use of day care and short-stay respite care, and the use of visiting services might be effective for patients who require the level of long-term care $2-3$.

One of the contributors to the heavier time-dependent burden on younger caregivers than older caregivers in the present study might be employment status (Table 2). An Iranian cross-sectional study showed that employed caregivers had a remarkably higher self-perceived burden than retired caregivers, suggesting that employment status affected the burden of caregivers[24]. In the present study, younger employed caregivers probably had longer working hours and, therefore, experienced greater difficulty in sparing time required for the care of the patient than older caregivers, 
leading to a high time-dependent burden. Another contributor to the high timedependent burden on younger caregivers maybe household work because younger caregivers have a larger amount of household work than older caregivers[25]. To ease the time-dependent burden on younger caregivers, the Family Medical Leave Act in the US[26] has provided care services according to the caregiver's burden status. In contrast, the Japanese long-term care system has provided care services based on the patient's condition, not the caregiver's burden status. To ameliorate the imbalance between the demand and supply of care services, the Japanese long-term care system should provide care services according to both the patient's condition and the caregivers' time-dependent burden.

The present study has several limitations. First, because the self-perceived burden of the family caregivers in the present cross-sectional study was assessed after the patients, who they cared for, died, a theory-based recall bias might affect the selfperceived burden of the family caregivers[27]. Caregivers with a tendency to believe that they had a burden during the home care period were likely to overestimate their past burden in a retrospective survey. A real-time self-perceived burden on family caregivers during the home care period should be strictly assessed. Second, the trajectories of the family caregivers' burden and the patients' care-needs level were not 
included in the present study because of the nature of its cross-sectional study design. A longitudinal study design should be used to estimate the association more precisely. Third, the present study mainly included patients with moderate to severe care needs levels [requiring long-term care $2-5, \mathrm{n}=268(78.4 \%)$ ]. Although the time-dependent burden was comparable between mild and severe care needs levels [adjusted odds ratio of care needs level 1 vs. 3, $1.21(0.48-2.93)]$, their clinical impact should be verified in a larger cohort.

In conclusion, the present study identified the time-dependent burden as the major burden on the family caregivers of patients with cancer and clarified the nonlinear association between the care needs level and the time-dependent burden, suggesting an imbalance between care needs and care supply in the Japanese long-term care system. To develop a practical long-term care system, long-term care should be provided to each patient according to the clinical characteristics of both the patients and their family caregivers.

\section{Declarations}


Funding support: This study was conducted as a specific research of the JHOPE4

study funded by the Japan Hospice Palliative Care Foundation and JSPS KAKENHI

Grant Number JP18J225780.

Conflict of interest disclosures: The authors declare no conflict of interest.

Availability of data: The data were collected by questionnaire with the approval of the

Ethics Committee of Tohoku University.

Code availability: N/A

Ethics approval: The study protocol of JHOPE was approved by the ethics committee of Tohoku University Hospital and all participating institutions, and the protocol of the present study was approved by the Osaka University Hospital.

All family caregivers gave informed consent to JHOPE4.

Consent for participation and publication: Informed consent was given to all family caregivers for participation in JHOPE4 and possible publication of the results. 


\section{References}

1. Joshua L (2017) Aging and Long Term Care Systems: A Review of Finance and Governance Arrangements in Europe, North America and Asia-Pacific. Soc Prot Labor Discuss Pap 1-112

2. Otsuki N, Fukui S, Nakatani E (2020) Quality and cost-effectiveness analyses of home-visit nursing based on the frequency of nursing care visits and patients' quality of life: A pilot study. Geriatr Gerontol Int 20:36-41. https://doi.org/10.1111/ggi.13816

3. Johansen S, Cvancarova M, Ruland C (2018) The Effect of Cancer Patients' and Their Family Caregivers' Physical and Emotional Symptoms on Caregiver Burden. Cancer Nurs 41:91-99. https://doi.org/10.1097/NCC.0000000000000493

4. Matsuda S, Yamamoto M (2001) Long-term care insurance and integrated care for the aged in Japan. Int J Integr Care 1:1-11. https://doi.org/10.5334/ijic.39

5. Tsutsui T, Muramatsu N (2005) Care-needs certification in the long-term care insurance system of Japan. J Am Geriatr Soc 53:522-527. https://doi.org/10.1111/j.1532-5415.2005.53175.x 
6. Morita T, Miyashita M, Yamagishi A, et al (2013) Effects of a programme of interventions on regional comprehensive palliative care for patients with cancer: A mixed-methods study. Lancet Oncol 14:638-646. https://doi.org/10.1016/S1470-2045(13)70127-X

7. Schulz R, Beach SR (1999) Caregiving as a risk factor for mortality: The caregiver health effects study. J Am Med Assoc 282:2215-2219. https://doi.org/10.1001/jama.282.23.2215

8. Franchini L, Ercolani G, Ostan R, et al (2020) Caregivers in home palliative care: gender, psychological aspects, and patient's functional status as main predictors for their quality of life. Support Care Cancer 28:3227-3235. https://doi.org/10.1007/s00520-019-05155-8

9. Given CW, Given B, Stommel M, et al (1992) The caregiver reaction assessment (CRA) for caregivers to persons with chronic physical and mental impairments. Res Nurs Health 15:271-283. https://doi.org/10.1002/nur.4770150406

10. Milbury K, Badr H, Fossella F, et al (2013) Longitudinal associations between caregiver burden and patient and spouse distress in couples coping with lung cancer. Support Care Cancer 21:2371-2379. https://doi.org/10.1007/s00520-013$1795-6$ 
11. Lee YH, Liao YC, Shun SC, et al (2018) Trajectories of caregiver burden and related factors in family caregivers of patients with lung cancer. Psychooncology 27:1493-1500. https://doi.org/10.1002/pon.4678

12. Hsu T, Loscalzo M, Ramani R, et al (2014) Factors associated with high burden in caregivers of older adults with cancer. Cancer 120:2927-2935.

https://doi.org/10.1002/cncr.28765

13. Wen FH, Chen JS, Chou WC, et al (2019) Family Caregivers' Subjective Caregiving Burden, Quality of Life, and Depressive Symptoms Are Associated With Terminally Ill Cancer Patients’ Distinct Patterns of Conjoint Symptom Distress and Functional Impairment in Their Last Six Months of Life. J Pain Symptom Manage 57:64-72. https://doi.org/10.1016/j.jpainsymman.2018.09.009

14. Masukawa K, Aoyama M, Morita T, et al (2018) The Japan hospice and palliative evaluation study 4: A cross-sectional questionnaire survey. BMC Palliat Care 17:1-7. https://doi.org/10.1186/s12904-018-0319-z

15. Amano K, Maeda I, Morita T, et al (2017) C-reactive protein, symptoms and activity of daily living in patients with advanced cancer receiving palliative care. J Cachexia Sarcopenia Muscle 8:457-465. https://doi.org/10.1002/jcsm.12184 
16. Jin X, Mori T, Sato M, et al (2020) Individual and regional determinants of longterm care expenditure in Japan: evidence from national long-term care claims.

Eur J Public Health 1-6. https://doi.org/10.1093/eurpub/ckaa065

17. Lin HR, Otsubo T, Imanaka Y (2015) The effects of dementia and long-term care services on the deterioration of care-needs levels of the elderly in Japan. Med (United States) 94:e525. https://doi.org/10.1097/MD.0000000000000525

18. Shimizutani S (2014) The Future of Long-term Care in Japan. Asia-Pacific Rev 21:88-119. https://doi.org/10.1080/13439006.2014.925199

19. Matsuda T, Iwagami M, Suzuki T, et al (2019) Correlation between the Barthel Index and care need levels in the Japanese long-term care insurance system.

Geriatr Gerontol Int 19:1186-1187. https://doi.org/10.1111/ggi.13777

20. Miyashita M, Yamaguchi A, Kayama M, et al (2006) Validation of the Burden Index of Caregivers (BIC), a multidimensional short care burden scale from Japan. Health Qual Life Outcomes 4:1-9. https://doi.org/10.1186/1477-7525-452

21. Ge L, Mordiffi SZ (2017) Factors Associated with Higher Caregiver Burden among Family Caregivers of Elderly Cancer Patients: A Systematic Review. Cancer Nurs 40:471-478. https://doi.org/10.1097/NCC.0000000000000445 
22. Rha SY, Park Y, Song SK, et al (2015) Caregiving burden and the quality of life of family caregivers of cancer patients: The relationship and correlates. Eur J Oncol Nurs 19:376-382. https://doi.org/10.1016/j.ejon.2015.01.004

23. Igarashi A, Yamamoto-Mitani N, Yoshie S, Iijima K (2017) Patterns of longterm care services use in a suburban municipality of Japan: a population-based study. Geriatr Gerontol Int 17:753-759. https://doi.org/10.1111/ggi.12781

24. Vahidi M, Mahdavi N, Asghari E, et al (2016) Other Side of Breast Cancer: Factors Associated with Caregiver Burden. Asian Nurs Res (Korean Soc Nurs Sci) 10:201-206. https://doi.org/10.1016/j.anr.2016.06.002

25. Mor V, Allen S, Malin M (1994) The psychosocial impact of cancer on older versus younger patients and their families. Cancer. https://doi.org/10.1002/10970142(19941001)74:7+<2118::AID-CNCR2820741720>3.0.CO;2-N

26. Longacre ML, Valdmanis VG, Handorf EA, Fang CY (2017) Work impact and emotional stress among informal caregivers for older adults. Journals Gerontol Ser B Psychol Sci Soc Sci 72:522-531. https://doi.org/10.1093/geronb/gbw027

27. Smith D, Loewenstein G, Jepson C, et al (2008) Mispredicting and Misremembering: Patients With Renal Failure Overestimate Improvements in 
Quality of Life After a Kidney Transplant. Heal Psychol 27:653-658.

https://doi.org/10.1037/a0012647 
Table 1. Participants' characteristics stratified by care needs levels

\begin{tabular}{|c|c|c|c|c|}
\hline & \multicolumn{4}{|c|}{ Care needs level* $^{*}$} \\
\hline & None & Mild & Moderate & Severe \\
\hline Number & 22 & 52 & 125 & 143 \\
\hline Age of patients, year & $73(59-80)$ & $76(70-83)$ & $78(68-87)$ & $76(68-84)$ \\
\hline Male patients, n (\%) & $15(68.2)$ & $32(61.5)$ & $72(57.6)$ & $93(65.0)$ \\
\hline Age of family caregivers, year & $65 \pm 11$ & $65 \pm 12$ & $62 \pm 12$ & $62 \pm 12$ \\
\hline Male family caregivers, n (\%) & $6(27.3)$ & $18(34.6)$ & $32(25.6)$ & $32(22.4)$ \\
\hline \multicolumn{5}{|l|}{ Type of cancer, n (\%) } \\
\hline Lung & $4(18.2)$ & $13(25.0)$ & $19(15.2)$ & $31(21.7)$ \\
\hline Upper and lower gastrointestinal tracts & $6(27.3)$ & $14(26.9)$ & $28(22.4)$ & $34(23.8)$ \\
\hline Liver, biliary system, and pancreas & $8(36.4)$ & $12(23.1)$ & $33(26.4)$ & $26(18.2)$ \\
\hline Others & $4(18.2)$ & $13(25.0)$ & $45(36.0)$ & $52(36.4)$ \\
\hline \multicolumn{5}{|l|}{ Caregiver's relationship with patient, n (\%) } \\
\hline Spouse & $13(59.1)$ & $31(59.6)$ & $57(45.6)$ & $72(50.3)$ \\
\hline Child & $5(22.7)$ & $18(34.6)$ & $53(42.4)$ & $60(42.0)$ \\
\hline Son/daughter in law & $1(4.5)$ & $2(3.8)$ & $9(7.2)$ & $5(3.5)$ \\
\hline Parents & $0(0.0)$ & $1(1.9)$ & $1(0.8)$ & $0(0.0)$ \\
\hline Siblings & $2(9.1)$ & $0(0.0)$ & $4(3.2)$ & $2(1.4)$ \\
\hline Others & $1(4.5)$ & $0(0.0)$ & $1(0.8)$ & $4(2.8)$ \\
\hline Home care period, months & $0.5(0.3-1.8)$ & $1.2(0.6-2.5)$ & $1.4(0.7-3.0)$ & $1.1(0.6-3.0)$ \\
\hline $\begin{array}{l}\text { The period from the date of death to date } \\
\text { of response, months }\end{array}$ & $14.4(9.0-22.3)$ & $15.4(9.7-23.1)$ & $13.8(11.0-19.3)$ & $16.0(10.4-24.9)$ \\
\hline Cluster 1, n (\%) & $13(59.1)$ & $27(51.9)$ & $68(54.4)$ & $67(46.9)$ \\
\hline 2 & $9(40.9)$ & $25(48.1)$ & $57(45.6)$ & $76(53.1)$ \\
\hline Mental condition of family caregiver & $2(1.3-3.0)$ & $2(2.0-3.0)$ & $2(2.0-3.0)$ & $2(1.5-3.0)$ \\
\hline \multicolumn{5}{|l|}{ Burden Index of Caregiver, domains } \\
\hline Time-dependent burden & $2.5(2.0-4.0)$ & $2.0(2.0-4.0)$ & $4.0(2.0-5.0)$ & $3.0(2.0-4.0)$ \\
\hline Emotional burden & $0.5(0.0-2.0)$ & $1.0(0.0-2.0)$ & $1.0(0.0-2.0)$ & $1.0(0.0-2.0)$ \\
\hline Spiritual burden & $1.0(0.0-2.0)$ & $1.5(0.0-2.0)$ & $2.0(0.0-2.0)$ & $1.0(0.0-2.0)$ \\
\hline Physical burden & $1.5(0.0-2.0)$ & $2.0(0.0-3.0)$ & $2.0(0.0-3.0)$ & $1.0(0.0-3.0)$ \\
\hline Service burden & $1.5(0.0-2.0)$ & $1.0(0.0-2.0)$ & $1.0(0.0-2.0)$ & $1.0(0.0-2.0)$ \\
\hline
\end{tabular}

Mean \pm standard deviation; median $(25 \%-75 \%)$

$P \geq 0.05$ for all variables

*Mild care needs level included requiring support 1, requiring support 2 and requiring long-term care 1 . Moderate care needs level included requiring long-term care 2 and 
requiring long-term care 3. Severe care needs level included requiring long-term care 4 and requiring long-term care 5. 
Table 2. Contributors to time-dependent burden of caregivers

\begin{tabular}{|c|c|c|c|c|}
\hline & \multicolumn{2}{|c|}{ Unadjusted Model } & \multicolumn{2}{|c|}{ Adjusted Model* } \\
\hline & OR $(95 \% \mathrm{CI})$ & $\mathrm{P}$ & OR $(95 \% \mathrm{CI})$ & $\mathrm{P}$ \\
\hline \multicolumn{5}{|l|}{ Age of patient } \\
\hline$<65$ & $0.47(0.18-1.11)$ & 0.096 & $0.21(0.05-0.80)$ & 0.026 \\
\hline $65-74$ & $0.78(0.39-1.55)$ & 0.478 & $0.65(0.23-1.78)$ & 0.400 \\
\hline $75-84$ & $1.14(0.59-2.21)$ & 0.693 & $1.00(0.43-2.35)$ & 0.995 \\
\hline$\geq 85$ & 1.00 (reference) & & 1.00 (reference) & \\
\hline Female patient & $1.60(0.95-2.67)$ & 0.074 & $2.01(0.98-4.35)$ & 0.061 \\
\hline \multicolumn{5}{|l|}{ Age of family caregiver } \\
\hline$<55$ & $3.00(1.28-7.67)$ & 0.015 & $4.91(1.13-22.49)$ & 0.036 \\
\hline $55-64$ & $1.82(0.79-4.63)$ & 0.180 & $2.96(0.89-10.45)$ & 0.082 \\
\hline $65-74$ & $1.33(0.56-3.42)$ & 0.539 & $1.74(0.62-5.25)$ & 0.305 \\
\hline$\geq 75$ & 1.00 (reference) & & 1.00 (reference) & \\
\hline Female family caregiver & $1.57(0.86-3.02)$ & 0.156 & $1.85(0.85-4.22)$ & 0.132 \\
\hline \multicolumn{5}{|l|}{ Care needs level } \\
\hline None & $0.43(0.07-1.60)$ & 0.274 & $0.50(0.07-2.12)$ & 0.404 \\
\hline Mild & $1.02(0.44-2.24)$ & 0.956 & $1.08(0.43-2.57)$ & 0.863 \\
\hline Moderate & $1.88(1.07-3.33)$ & 0.029 & $1.87(1.01-3.52)$ & 0.048 \\
\hline Severe & 1.00 (reference) & & 1.00 (reference) & \\
\hline Home care period, per 4 weeks & $1.00(0.94-1.05)$ & 0.958 & $0.98(0.91-1.03)$ & 0.513 \\
\hline
\end{tabular}

$\mathrm{CI}$, confidence interval; OR, odds ratio

*Adjusting for age of patient (year), age of family member (year), patient and family member sex, type of cancer (lung; upper and lower gastrointestinal tracts; liver, biliary system, and pancreas; and others) in the patient, family member's relationship with patient (spouse, child, son/daughter in law, parents; siblings, and others), mental status of family members, home care period (week), the period from the date of death to date of response (month), and clusters (1 and 2). 


\section{Figure legends}

Figure 1. Flow diagram of inclusion and exclusion of study participants. JHOPE4, Japan Hospice and Palliative Evaluation study 4.

Figure 2. Proportion of the answers to each BIC question. BIC, Burden Index of

Caregivers.

Figure 3. Proportion of the answers to BIC question 1 and 2 in each care needs level.

BIC, Burden Index of Caregivers. 


\section{Figure 1}

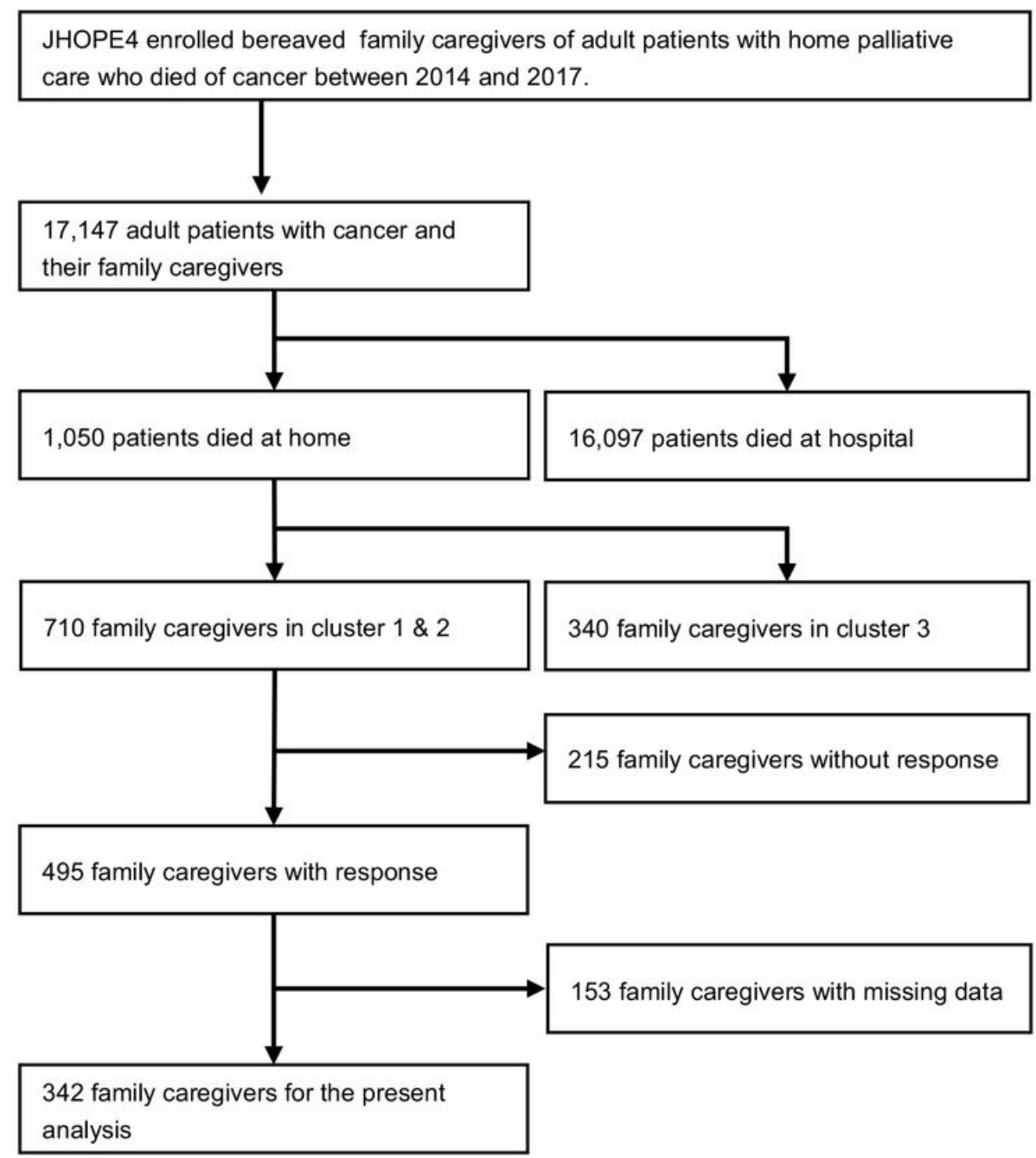

\section{Figure 1}

Flow diagram of inclusion and exclusion of study participants. JHOPE4, Japan Hospice and Palliative Evaluation study 4. 


\section{Figure 2}

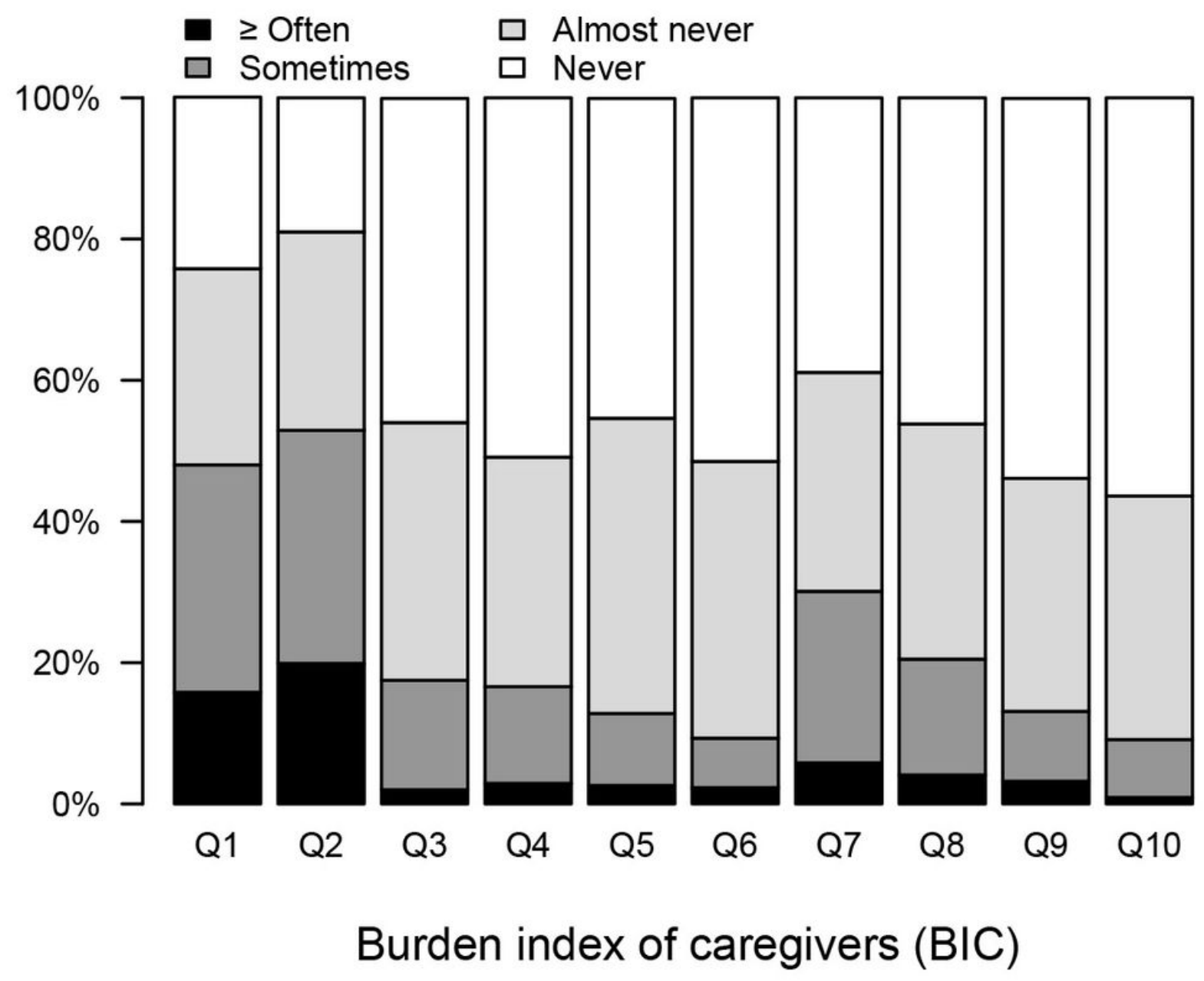

Figure 2

Proportion of the answers to each BIC question. BIC, Burden Index of Caregivers. 
Figure 3

a. BIC 1 "I cannot freely leave the house because of caregiving." b. BIC 2 "I do not have enough time for myself because of caregiving." c. Time-dependent burden (BIC $1+$ BIC 2)

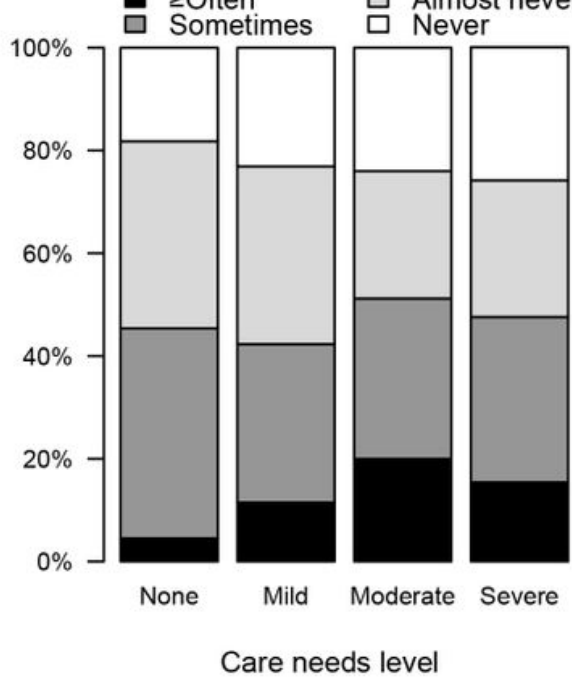

- $\mathrm{BIC} 1 \geq$ often and/or BIC $2 \geq$ often $\square$ BIC $1<$ often and $\mathrm{BIC} 2<$ often
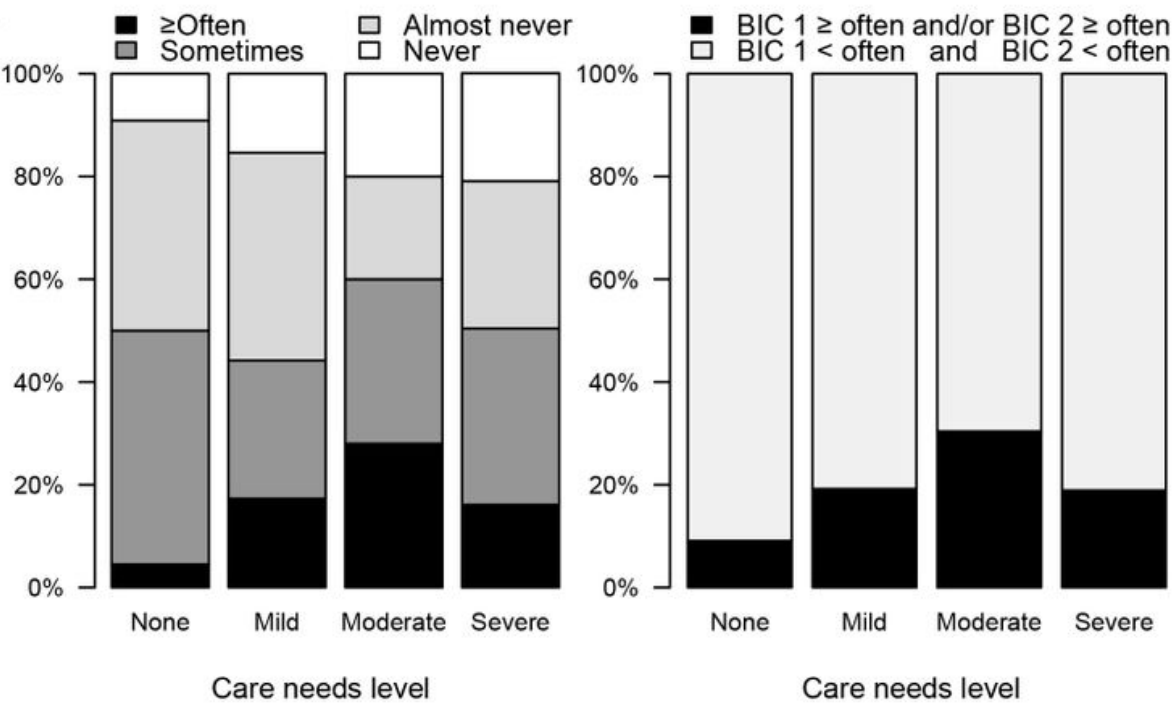

Figure 3

Proportion of the answers to BIC question 1 and 2 in each care needs level. BIC, Burden Index of Caregivers.

\section{Supplementary Files}

This is a list of supplementary files associated with this preprint. Click to download.

- coidisclosure.pdf

- supplementarytable1.pdf

- supplementarytable2.pdf 